\title{
Diffusion Weighted Imaging in Magnetic Resonance Imaging for Prostate Cancer Diagnosis: Current Efficiency as a Standalone Sequence for an Unenhanced MRI Experience - a Pilot Study
}

\author{
Nechifor-Boilă IA ${ }^{1,2}$, Borda Angela ${ }^{3}$, Loghin Andrada ${ }^{3}$, Nechifor-Boilă Adela ${ }^{3}$, Chibelean C 2 \\ Department of Anatomy, Târgu-Mureș University of Medicine and Pharmacy, Târgu-Mureș, Romania \\ 2 Department of Urology, Târgu-Mureș University of Medicine and Pharmacy, Romania \\ Department of Histology, Târgu- Mureș University of Medicine and Pharmacy, Romania
}

\begin{abstract}
Objective: Diffusion Weighted Imaging (DWI) is the main sequence in the multiparametric prostate MRI protocol together with T2 and dynamic contrast-enhanced T1, leading to detection rates up to $60 \%$ in prostate cancer diagnosis. However, the use of intravenous contrast can have severe side-effects, making the use of unenhanced MRI sequences essential. The aim of our study was to assess the feasibility and efficiency of DWI as a standalone MRI technique for prostate cancer diagnosis. Methods: We performed a prospective cohort study at our department (09.2014-05.2015) and formed a study lot consisting in five prostate cancer patients that were scheduled for radical prostatectomy. Multiparametric MRI was performed (with DWI and T2 sequences) and the images were interpreted according to the PI-RADS system. The final histopathological result after prostatectomy served as gold standard. Results: A series of 9 lesions were detected and analyzed on DWI. At qualitative interpretation, DWI had a sensitivity of $85.7 \%$ and a specificity of $50 \%$. The corresponding positive and negative likelihood ratios were 1.71 and 0.286 , respectively $(p=0.417)$. ADC analysis revealed a mean value of $1.2^{*} 10-3 \mathrm{~mm} 2 / \mathrm{s}$ for the benign lesions while the corresponding value was 0.8*10-3 for the malignant ones, regardless of tumor size and Gleason scoring. Conclusion: DWI is a feasible technique in the current clinical environment, with a good sensitivity and a medium specificity. Furthermore, an association to the anatomical T2 sequence could enhance the diagnostic efficiency of DWI and should be assessed in larger studies.
\end{abstract}

Keywords: DWI, prostate, multiparametric MRI, sensitivity, specificity

Received: 15 August 2015 / Accepted: 03 September 2015

\section{Introduction}

Multiparametric MRI (MpMRI) of the prostate is nowadays the most accurate imaging technique for Prostate Cancer (PC) diagnosis, with detection rates up to $60 \%$. In contrast, the classical diagnostic triad composed by the low specificity PSA, the low sensitivity Digital Rectal Examination and the histopathological diagnosis of prostate biopsy have overall detection rates up to $40 \%$ [1].

The basic MpMRI protocol, according to the second version of the Prostate Imaging and Report and Data System (PI-RADS), should be composed by three basic sequences: T2, Diffusion Weighted Imaging (DWI) and Dynamic Contrast-Enhanced T1 (DCE), with DWI having the predominant role, followed by T2. DCE has more of an orientating role, being used mainly when in doubt [2].The use of intravenous gadolinium in DCE can have its setbacks, mainly in the chronic renal failure patients where, for uncertain reasons, there is a risk for a severe disease called Nephrogenic Systemic Fibrosis [3].A potential solution could be the use of sequences as standalone techniques for asuperior native non-contrast -enhanced MpMRI. The main candidate is DWI, with its assessment of water diffusion (and thus cellularity) and also its pre-

* Correspondence to: Andrada Loghin

E-mail: andradaloghin@yahoo.com disposition to artifacts (by movement or eddy currents) requiring special examination techniques like the use of an endorectal coil for $1.5 \mathrm{~T}$ scanners $[4,5]$. However, this can cause discomfort for the patient, increase movement artifacts and influence interpretation. The aim of our pilot study was to assess the feasibility and efficiency of DWI at 1.5T without the endorectal coil for PC diagnosis.

\section{Methods}

Inclusion protocol

We performed a prospective, single-cohort trial at our department, for the period 09.2014-05.2015. The target population was biopsy-proven prostate cancer patients that were not subjected to MpMRI before the intervention. After having obtained the approval of our local ethics committee, we started building the study lot based upon a series of strict inclusion and exclusion criteria.

We included males above the age of 18 , having a clinical suspicion of PC (abnormal PSA levels or DRE), that was later confirmed by prostate biopsy and were scheduled for radical prostatectomy. Additionally, there was an agreed 1 month interval between the date of biopsy and the MpMRI exam in order to avoid biopsy-related artifacts like hematomas.

Patients having less than 1 month after biopsy, with previous diagnosis of other prostatic diseases (like chronic 
prostatitis) or factors incompatible with a MRI examination (like implants,pacemakers, etc) were excluded from the trial.

\section{MRI examination}

The MRI examinations were performed on a General Electric Signa Excite II 1.5T scanner (GE medical systems) using a standard, 8 channel high-resolution abdominal coil with the patient in supine position. All images were obtained in the axial plane of the prostate.

The T2 images were obtained using a fast relaxation Fast Spin Echo (frFSE) sequence, with TR 2400ms, TE 88.9 $\mathrm{ms}$, flip angle $90^{\circ}, 3 \mathrm{~mm}$ thickness, $0 \mathrm{~mm}$ gaps, a scan matrix of 256/224, NEX 4, FOV $180 \mathrm{~mm}$.

The DWI images were obtained at three b-values ( 0 , 100 and $1000 \mathrm{~s} / \mathrm{mm}^{2}$ ) using a standard Spin Echo - Echo Planar Sequence (SE-EPI) with FOV 32/25.6, with TR $4500 \mathrm{~ms}$, TE $83.8 \mathrm{~ms}$, a scan matrix of $96 / 128$, NEX 12 , flip angle $90^{\circ}$.

\section{Interpretation protocol}

All mpMRI images were examined off-line using a DICOM viewer (RadiANTDicom viewer, Medixant Inc.). Images were interpreted in a blind manner by a team formed by a radiologist and an urologist. For DWI, interpretation was performed both qualitatively and quantitatively by the means of the ADC. The qualitative interpretation was performed by direct visual assessment of the suspicious lesion, classifying it in the proper PI-RADS category (Table I).

Table I. PI-RADS assessment categories and their clinical significance according to the ESUR guidelines version 2.0

\begin{tabular}{|c|c|}
\hline $\begin{array}{l}\text { PI-RADS } \\
\text { categories }\end{array}$ & Classification \\
\hline PI-RADS 1 & $\begin{array}{l}\text { Very low (clinically significant cancer is highly unlikely to be } \\
\text { present) }\end{array}$ \\
\hline PI-RADS 2 & Low (clinically significant cancer is unlikely to be present) \\
\hline PI-RADS 3 & $\begin{array}{l}\text { Intermediate (the presence of clinically significant cancer is } \\
\text { equivocal) }\end{array}$ \\
\hline PI-RADS 4 & High (clinically significant cancer is likely to be present) \\
\hline PI-RADS 5 & $\begin{array}{l}\text { Very high (clinically significant cancer is highly likely to be } \\
\text { present) }\end{array}$ \\
\hline
\end{tabular}

After the qualitative interpretation, ADCs were calculated for all suspicious lesions using the following formula:

$$
A D C=-\frac{1}{b} \ln \left(\frac{S_{1000}}{S_{0}}\right)
$$

where $\mathrm{b}$ stands for the $\mathrm{b}$-value of the DWI sequence (i.e. $b=1000 \mathrm{~s} / \mathrm{mm}^{2}$ in our study) and $S 1000$ and $S 0$ are the lesion signal intensities measured on the diffusion-weighted image $(b=1000 \mathrm{~s} / \mathrm{mm} 2)$ and the reference one $(b=0 \mathrm{~s} /$ $\mathrm{mm} 2$ ), respectively. The signal intensities (mean pixel intensities) were obtained by drawing Regions of Interest (ROIs) over the lesions at their maximum intensity on the axial images. ADC calculation was performed in the Excel
Software (Microsoft Office 2007, Microsoft, Redmond, USA) and all ADC values were inserted in the study database. For comparison purposes, the T2 images were interpreted separately. At the end of the interpretation session, all lesions were classified using the PI-RADS criteria [2].

\section{The gold standard}

In our study, the gold standard was represented by the result of the pathological exam from the radical prostatectomy specimen. Detailed histopathological reports were obtained for each patients, including the zonal sketches made by the pathologist in order to clearly localize the tumor(s).

\section{Statistical Analyses}

We analyzed the data using the EpiInfo v 7.1.4.0 (Centers for Disease Control and Prevention, Atlanta, USA) and the IBM-SPSS V19.0 demo (SPSS Inc, an IBM company) software. After dichotomization of the initial PI-RADS classification as benign $(1,2)$ and malignant $(3,4,5)$, clinical efficiency indicators (sensitivity, specificity, positive and negative likelihood ratio) were calculated for qualitative DWI, referring to the gold standard. ADC values were analyzed according to the final diagnosis.

\section{Ethical issues}

Approval from the local ethics committee was obtained before the study was started. Before entering the study, each patient was briefed by the investigator concerning his medical condition, the particularities of the clinical trial and had to sign an informed consent form. All data concerning the patients is considered confidential and it was used only by the study team in a secure database.

\section{Results}

During the selection period, a total of 21 patients were considered eligible for the trial. After applying the inclusion and exclusion criteria, 11 were considered unsuitable for different reasons: patients unfit for surgery/MRI because of their co-morbidities, refusal to perform the MRI exam and various technical difficulties. Out of the remaining 9, two opted for oncological treatment and two had very poor quality MRI exams and were dismissed. Thus, the final lot consisted in a series of five biopsy-proven PC patients (summing up to 9 lesions) that were schedules for radical prostatectomy. Their ages ranged from 48 to 68 years, with a mean of 58.4. These patients had a clinical (abnormal DRE) and biochemical (high PSA values) suspicion of PC that was confirmed at biopsy. The mean PSA level was $6.22 \mathrm{ng} / \mathrm{dl}$ while the Gleason scores at biopsy were 6 in 2 cases and 7 in the remaining 3. Prostate size ranged between 30 and 45 , with an average of $36.2 \pm 5.67$ while the mean lesion size was $11.8 \pm 4 \mathrm{~mm}$.

At interpretation, quality scores were attributed for each examination, ranging from 1 (poor) to 4 (very good). Images were very good in 1 case, good in 3 cases and medium in the remaining one (Table II). 
Interpretation was performed blinded in 2 stages: first the T2 and afterwards DWI (both qualitative and quantitative-ADC). At qualitative interpretation, the given PIRADS scores between T2 and DWI images corresponded in 2 lesions, 4 were upgraded by DWI and the remaining 3 were downgraded. ADCs were calculated from the signal intensity levels on both $b=0 \mathrm{~s} / \mathrm{mm}^{2}$ and $b=1000 \mathrm{~s} /$ $\mathrm{mm}^{2}$ images. After applying the gold standard we found that 7 suspicious lesions were malignant while two were benign (benign prostatic hyperplasia). From the pathology reports, we found that 3 cases were staged pT2, one was pT3a and one was T1c, with a majority of Gleason 7 lesions (6 lesions). Detailed pathological information can be found in Table III.

After contingency table analysis we found a sensitivity of $85.7 \%$ and a specificity of $50 \%$. The corresponding positive and negative likelihood ratios were 1.71 and 0.286 ( $\mathrm{p}=0.417)$.

$\mathrm{ADC}$ analysis revealed a mean $\mathrm{ADC}$ of $1.2^{*} 10^{-3}$ for the benign lesions while for the malignant ones, the corresponding value was $0.8^{*} 10^{-3}$, regardless of tumor size and Gleason scoring.

\section{Discussion \\ Overview}

In this current study we tested the feasibility and efficiency of DWI as a standalone technique in our current clinical environment, using a $1.5 \mathrm{~T}$ scanner without an endorectal coil. Concerning lesion visibility we noted that all lesions were found on good and medium quality images, a feature that could have direct impact on lesion conspicuity and influence sensitivity [1]. However, several authors demonstrated that conspicuity is improved by using high $\mathrm{B}$ values like 1500 and $2000 \mathrm{~s} / \mathrm{mm}^{2}$ on 3T imagers, both on native and computed high b-valued images, aspect valid also for the $1.5 \mathrm{~T}$ machines $[6,7]$. Vural et al also suggest that even
Table II. Descriptive data concerning patient demographics, prostate size, lesion characteristics, and their interpretation using the PI-RADS system

\begin{tabular}{lc}
\hline Characteristic & Mean value \pm SD or N \\
\hline Age & $58.4 \pm 9.55$ \\
Prostate size & $36.2 \pm 5.67$ \\
PSA & $6.22 \pm 1.09$ \\
Lesion size & $11.3 \pm 4.12$ \\
Lesion topography & \\
- bilateral & 3 cases \\
-right lobe & 1 case \\
- left lobe & 1 case \\
Image quality & \\
- poor & 0 cases \\
- medium & 1 case \\
- good & 3 cases \\
- very good & 1 case \\
PI-RADS classification for DWI before dichotomi- & \\
sation & 1 lesion \\
- PI-RADS 2 & 3 lesions \\
- PI-RADS 3 & 5 lesions \\
- PI-RADS 4 & \\
PI-RADS classification for DWI after dichotomisa- & \\
tion & \\
- DWI "malignant" & 7 lesions \\
- DWI "benign" & 2 lesions \\
\hline
\end{tabular}

Table III. Histopathological characteristics of lesions after applying the gold standard

\begin{tabular}{lc}
\hline Characteristic & Mean value $\pm \mathrm{SD}$ or $\mathrm{N}$ \\
\hline Final histopathological results & \\
- malignant (prostatic adenocarcinoma) & 7 lesions \\
- benign (focus of benign prostatic hyperplasia) & 2 lesions \\
Gleason scores & \\
- Gleason $4+3=7$ & 6 lesions \\
- Gleason $3+3=6$ & 1 lesions \\
Presence of Extracapsular Extension & 1 lesion \\
Affected prostatic lobe & \\
- bilateral & 3 cases \\
- right lobe & 1 case \\
- left lobe & 1 case \\
\hline
\end{tabular}

higher computed $\mathrm{b}$ values could be obtained (reaching $3000 \mathrm{~s} / \mathrm{mm}^{2}$ ) and still preserve the overall detection rates as for the native low b-valued ones [8].
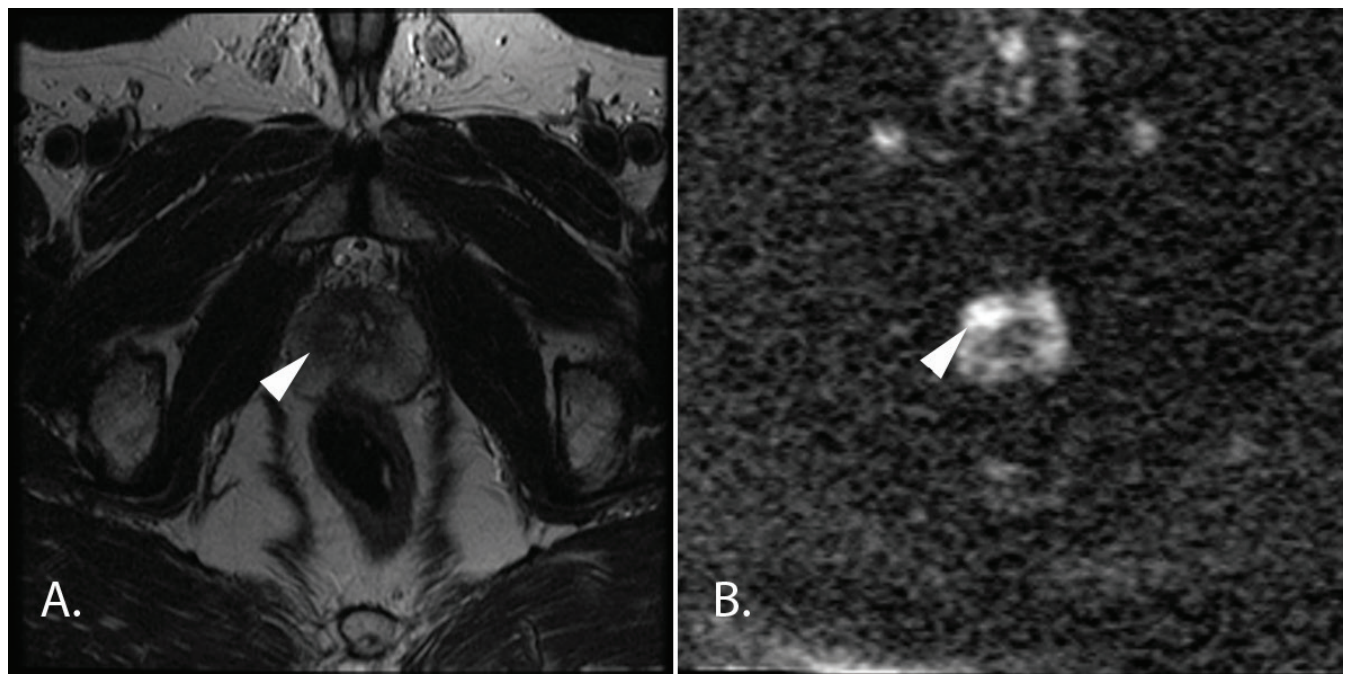

Fig. 1. Multiparametric MRI of the prostate in a 49-year-old prostate cancer patient (PSA $5 \mathrm{ng} / \mathrm{ml}$, a right-sided cancer with a Gleason score of $3+4=7$ at biopsy). The target lesion (arrow head) has a discrete hyposignal in T2 (A) and a moderately increased signal intensity in DWI (B), suggestive of malignancy. After radical prostatectomy, the histopathological examination of the prostate identified this lesion as the index lesion (an overall volume of $2.4 \mathrm{cc}$ and a Gleason score identical to the one obtained at biopsy). 


\section{Diagnostic efficiency}

At interpretation, all lesions were classified according to the PI-RADS system and dichotomized at the value of 2 . Thus, we considered all lesions in the 1 and 2 classes as benign and the remaining 3,4, and 5 as malignant. For qualitative DWI we obtained a good sensitivity (85.7\%) with a low specificity (50\%). Our results are different from those of Kitamura et al who found lower sensitivity rates for DWI (51\%) and a higher specificity (89\%). However, their results were also influenced by the quantitative interpretation $(\mathrm{ADC})$ and the $\mathrm{ADC}$ maps[4].

Concerning the ADC values, our results are in accordance with those obtained by deCobelli et al who also demonstrated a stratified decrease in ADC values in accordance to the increase in Gleason scoring for malignant lesions [9].

In the case of a PC patient with extracapsular extension (ECE), we obtained a "malignant" ADC value of $0.67^{*} 10^{-}$ ${ }^{3} \mathrm{~s} / \mathrm{mm} 2$, which is in agreement with the study of Woo et al that found there is no significant difference between ADC values between patients with and without ECE for a Gleason score higher than 4 (a Gleason score of 7 in our case) [10].

\section{Further improvements}

The main limit of our current study is the small number of cases, restricting the possible interpretation scenarios to one (malignant). In order to fully explore the potential of DWI larger case series are necessary with the inclusion of patients with other prostatic pathology (e.g. benign prostatic hyperplasia). The specificity of DWI could be improved by the association with an anatomical sequence like T2 with or without the use of fused images, resulting in a more effective MRI protocol [11].

\section{Conclusion}

DWI is a feasible technique in the current clinical environment, with a good sensitivity and a medium specificity. Furthermore, an association to the anatomical T2 sequence could enhance the diagnostic efficiency of DWI and should be assessed in larger studies.

\section{Conflicts of interest statement}

There are no conflicts of interest.

\section{Acknowledgement}

This paper is supported by the Sectoral Operational Programme Human Resources Development (SOP HRD), financed from the European Social Fund and by the Romanian Government under the contract number POSDRU/159/1.5/S/133377/.

\section{References}

1. Lim KS, Tan $\mathrm{CH}$. Diffusion-weighted MRI of adult male pelvic cancers. Clin Radiol. 2012,67(9):899-908.

2. Prostate Imaging and Report and Data system: Version 2. 2015.

3. Thomsen HS, Morcos SK, Almen T, et al. Nephrogenic systemic fibrosis and gadolinium-based contrast media: updated ESUR Contrast Medium Safety Committee guidelines. Eur Radiol. 2013;23(2):307-318.

4. Kitamura K, Muto S, Yokota I, et al. Feasibility of multiparametric prostate magnetic resonance imaging in the detection of cancer distribution: histopathological correlation with prostatectomy specimens. Prostate Int. 2014;2(4):188-195.

5. Bittencourt LK, Hausmann D, Sabaneeff N, et al. Multiparametric magnetic resonance imaging of the prostate: current concepts. Radiol Bras. 2014;47(5):292-300.

6. Grant KB, Agarwal HK, Shih $\mathrm{JH}$, et al. Comparison of calculated and acquired high b value diffusion-weighted imaging in prostate cancer. Abdom Imaging. 2015;40(3):578-586.

7. Rosenkrantz AB, Hindman N, Lim RP, et al. Diffusion-weighted imaging of the prostate: Comparison of b1000 and b2000 image sets for index lesion detection. J Magn Reson Imaging. 2013;38(3):694-700.

8. Vural M, Ertas G, Onay A, et al. Conspicuity of peripheral zone prostate cancer on computed diffusion-weighted imaging: comparison of cDWl1500, cDWI2000, and cDWI3000. Biomed Res Int. 2014;2014:768291.doi:10.1155/2014/768291.

9. De CF, Ravelli S, Esposito A, et al. Apparent diffusion coefficient value and ratio as noninvasive potential biomarkers to predict prostate cancer grading: comparison with prostate biopsy and radical prostatectomy specimen. AJR Am J Roentgenol. 2015;204(3):550-557.

10. Woo S, Cho JY, Kim SY, Kim SH. Extracapsular extension in prostate cancer: added value of diffusion-weighted $\mathrm{MRI}$ in patients with equivocal findings on T2-weighted imaging. AJR Am J Roentgenol. 2015;204(2):W168-W175.

11. Rosenkrantz AB, Mannelli L, Kong $X$, et al. Prostate cancer: utility of fusion of T2-weighted and high b-value diffusion-weighted images for peripheral zone tumor detection and localization. J Magn Reson Imaging. 2011;34(1):95-100. 